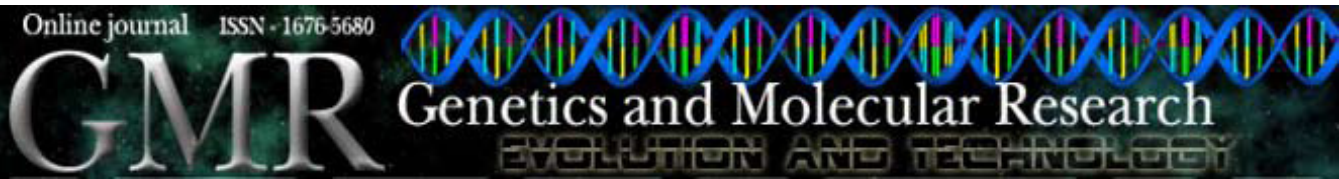

\title{
No association of polymorphisms in the suppressor of cytokine signaling (SOCS)-3 with rheumatoid arthritis in the Chinese Han population
} \author{
Jinan, PR China \\ Jinan, PR China \\ PR China \\ *These authors contributed equally to this study. \\ Corresponding author: Y.S. Wang \\ E-mail: sdjnwys@163.com / xfuli@163.com
}

L.P. Sun ${ }^{1,2 *}$, X.L. Ma ${ }^{2,3 *}$, H.X. Liu ${ }^{1}$, Y.S. Wang ${ }^{2,3}$ and X.F. Li ${ }^{1}$

${ }^{1}$ Department of Rheumatology, Qilu Hospital, Shandong University,

${ }^{2}$ Central Laboratory, Jinan Central Hospital Affiliated to Shandong University,

${ }^{3}$ Shandong Key Laboratory of Tumor Target Molecule, Shandong Province,

Genet. Mol. Res. 9 (3): 1518-1524 (2010)

Received May 10, 2010

Accepted June 5, 2010

Published August 3, 2010

DOI 10.4238/vol9-3gmr914

\begin{abstract}
Suppressor of cytokine signaling (SOCS)-3 is a key negative regulator of cytokine signaling that inhibits the JAK/STAT signal transduction pathway; there are reports describing its role in attenuating arthritis through SOCS-3 overexpression. We examined the relationship between polymorphisms in the coding sequence and promoter region of SOCS-3 and rheumatoid arthritis (RA) in a Chinese Han population. Two single-nucleotide polymorphisms in the SOCS3 5' region: $-1044 \mathrm{C}>\mathrm{A}$ within the promoter region and rs 12953258 $(-920 \mathrm{C}>\mathrm{A}$ ) in the 5'UTR (exon 2) of SOCS-3 were studied by restriction fragment length polymorphism analysis and tetra-ARMSPCR in 100 RA patients and 100 healthy adults. The prevalence of the homozygous genotype -1044 CC was $100 \%$ in both RA and control
\end{abstract}


groups. The heterozygous genotype (-920 C $>$ A) was present in $89 \%$ of RA and in $82 \%$ of the control group, which is significantly different from the distribution in Western people. There was no transmission disequilibrium between these two SNPs $\left(\mathrm{r}^{2}=0.000\right)$. We did not detect significant differences in allele or genotype frequencies for either of these SNPs between the RA group and controls $(\mathrm{P}>0.05)$. There was no association between rheumatoid factor and SOCS-3 SNP rs 12953258 $(\mathrm{P}=0.258)$. We conclude that SOCS-3 polymorphism is not a genetic risk factor for RA in Chinese patients.

Key words: Suppressor of cytokine signaling-3 (SOCS-3); Single-nucleotide polymorphism; Rheumatoid arthritis;

Chinese Han population

\section{INTRODUCTION}

Rheumatoid arthritis (RA) is a complex autoimmune disease resulting from sustained imbalance between pro- and anti-inflammatory mechanisms (Smolen and Steiner, 2003). Cytokines play an important role in the pathogenesis of a wide variety of inflammatory and autoimmune diseases, and investigations provide support for a critical role for cytokines in RA (Firestein and Zvaifler, 1997). Over the last decade, dramatic improvements in the treatment of RA have resulted from increased understanding of the role played by cytokines in the rheumatoid synovium. The development of the anti-tumor necrosis factor alpha (TNF- $\alpha$ ) agents infliximab (Maini et al., 1999), etanercept (Weinblatt et al., 1999) and adalimumab (Weinblatt et al., 2003) has been the most striking manifestation of this success, but other cytokines such as interleukin (IL)-6 and IL-1 have also been studied. It is important to recognize, however, that a sizeable proportion of patients do not respond to anti-TNF- $\alpha$ treatment (Maini et al., 1999; Weinblatt et al., 1999, 2003). For these patients, different therapeutic avenues must be explored. Therapeutic manipulation of endogenous-negative regulators of cytokine signaling could be a more effective therapeutic approach, as this may allow inhibition of multiple cytokines.

Suppressor of cytokine signaling (SOCS) belongs to the family of cytokine-inducible inhibitors of signaling including cytokine-inducible SH2 domain-containing protein and SOCS-1-7 (Starr et al., 1997). The SOCS proteins are key negative regulators of cytokine signaling that inhibit the JAK/STAT signal transduction pathway (Alexander, 2002). Transient SOCS expression can be induced by cytokines in a wide range of different tissues, and the tissues involved are different for different SOCS (Rottapel, 2001; Isomaki et al., 2007). Murine studies have suggested that RA would be characterized by reduced levels of SOCS-1 and/ or SOCS-3 in lymphocytes and synovial cells (Egan et al., 2003; Wong et al., 2006). Overexpression of SOCS-3 in joint macrophages and fibroblasts has been demonstrated to effectively block STAT3 activation during experimental arthritis and to attenuate disease severity (Shouda et al., 2001). Thus, SOCS-3 overexpression is effective in attenuating arthritis, but the role of endogenous SOCS expression and the mechanisms by which SOCS-3 overexpression suppresses arthritis have not been elucidated.

Given the importance of SOCS-3 in RA, it is necessary to know the genetic influences of the polymorphisms of SOCS-3 in RA. To test whether genetic variations of SOCS-3 contrib- 
ute to RA, this study was undertaken to see whether variations in the coding sequence (CDS) or promoter region of SOCS-3 are associated with RA in a Chinese Han population.

\section{MATERIAL AND METHODS}

\section{Subjects}

RA patients and controls were recruited from Qilu Hospital and Jinan Central Hospital affiliated with Shandong University. All patients met American College of Rheumatology (formerly, the American Rheumatism Association) 1987 revised criteria for the classification of RA (Arnett et al., 1988). Of the RA cases, 100\% (100/100 had longstanding disease, 82\% (82/100) were positive for rheumatoid factor and 78\% (78/100) were female. The group had a mean age at onset of 50.75 years. Control samples were taken from a pool of healthy individuals with no medical history of RA. A single control was matched to each case on the basis of gender, age ( \pm 5 years), and ethnicity (grandparental country/region of origin). All protocols and recruitment sites were approved by national and/or local institutional review boards, and informed written consent was obtained from all subjects. In total, the samples analyzed included 100 independent RA cases (78 women, 22 men) and 100 independent population controls ( 80 women, 20 men) of Shandong Province.

All blood samples were obtained with informed consent from Chinese Han individuals. The study protocol was reviewed and approved by the Chinese Ethics Committee of $\mathrm{Hu}-$ man Genetic Resources. Genomic DNA was extracted from blood leukocytes from all subjects, using standard protocols.

\section{Molecular genetic methods}

Primers were derived from a genomic sequence (GenBank accession No. NC_000017). We designed the following primer pairs: SOCS-3-o-F: 5' CCG CGC TCA GCC TTT CTC TGC TGC GA 3'; SOCS-3-o-R: 5' AGT CCA CAA AGG AGC CTT CGC GCG CG 3' (Hölter et al., 2007). Polymerase chain reaction (PCR) was performed according to standard protocols. The size of the PCR products was 338 bp. To genotype -1044 C >A, PCR with subsequent diagnostic restriction length polymorphism analyses was performed: the 338-bp SOCS-3 amplicon was digested with $M v a \mathrm{I}$ (NEB; C-allele: 45, 51, 67, and $175 \mathrm{bp}$, A-allele: 45, 51 and $242 \mathrm{bp}$ ). To genotype rs12953258 (-920 C $>$ A), we performed tetra-ARMS-PCR with other primers: SOCS-3-i-F: 5' CCC GCA CGC AGC CAG CCG TCC 3'; SOCS-3-i-R: 5' AGC TGG GCC GGG CGG GCG ACT 3'. Sizes of the PCR products were 338 bp (outer primer pair), $210 \mathrm{bp}$ (A-allele) and $170 \mathrm{bp}$ (C-allele). All PCR products were visualized on ethidium bromide-stained $2.5 \%$ agarose gels. Allele sizes were determined with a molecular weight standard (20-bp DNA Ladder Marker, Takara, Japan). Positive controls for the variant alleles were run on each gel. For validity of the genotypes, allele determinations were rated independently by at least two experienced individuals. Discrepancies were resolved unambiguously either by reaching consensus or by re-typing.

Selected samples with different genotypes were subject to direct sequencing for confirmation. The sequencing was performed using an ABI Prism3100 DNA sequencer (Applied Biosystems, Foster City, CA, USA). 


\section{Statistical analysis}

Analysis of linkage disequilibrium was performed using the SHEsis software package (Shi and He, 2005). A general linear model was calculated to analyze the effects of the polymorphism on continuous variables. Multiplicative interaction terms among the genotype, gender and rheumatism factor were used to analyze potential interactions of these variables and disease risk. SPSS for Windows version 10.0 was used for statistical analysis. We considered $\mathrm{P}$ values less than 0.05 statistically significant.

\section{RESULTS}

In the study groups comprising 100 RA (study group 1) and 100 healthy controls (study group 2), we detected two previously described single-nucleotide polymorphisms (SNPs): -1044 C>A within the promoter region and rs12953258 (-920 C >A) in the 5' untranslated region (5'UTR, exon 2) of SOCS-3.

Table 1 showed a haplotype frequency composed of $-920 \mathrm{C}>\mathrm{A}$ and $-1044 \mathrm{C}>\mathrm{A}$ of SOCS-3 in study subjects. There could be four haplotypes theoretically, but only two haplotypes, $-920 \mathrm{~A} /-1044 \mathrm{C}$ and $-920 \mathrm{C} /-1044 \mathrm{C}$, were detected whereas the $-920 \mathrm{~A} /-1044 \mathrm{~A}$ and $-920 \mathrm{C} /-1044 \mathrm{~A}$ haplotypes were not detected in any of the individuals examined in the current study. No differences in haplotype frequencies were detected for the two SNPs between 100 RA and 100 healthy controls $(\mathrm{P}=0.726)$.

\begin{tabular}{|c|c|c|c|c|c|}
\hline \multirow[t]{2}{*}{ Haplotype } & \multicolumn{2}{|c|}{ Chromosome numbers (2n) } & \multicolumn{2}{|c|}{ Haplotype frequency $(\%)$} & \multirow[t]{2}{*}{$\mathrm{P}$} \\
\hline & RA & Control & $\overline{\mathrm{RA}}$ & Control & \\
\hline$-920 \mathrm{~A} /-1044 \mathrm{C}$ & $105 / 200$ & $100 / 200$ & 52.5 & 50 & \\
\hline$-920 \mathrm{C} /-1044 \mathrm{C}$ & $95 / 200$ & $100 / 200$ & 47.5 & 50 & 0.726 \\
\hline$-920 \mathrm{~A} /-1044 \mathrm{~A}$ & $0 / 200$ & $0 / 200$ & 0 & 0 & \\
\hline$-920 \mathrm{C} /-1044 \mathrm{~A}$ & $0 / 200$ & $0 / 200$ & 0 & 0 & \\
\hline
\end{tabular}

$\mathrm{RA}=$ rheumatoid arthritis.

The linkage disequilibrium $\left(\mathrm{r}^{2}\right)$ between the two markers $(-1044 \mathrm{C}>\mathrm{A})$ and $\mathrm{rs} 12953258$ $(-920 \mathrm{C}>\mathrm{A})$ was 0.00 , implying that they are largely independently derived according to the SHEsis software (Shi and He, 2005). There was no linkage disequilibrium between the two SNPs. Differences in genotype frequencies were not detected for the two SNPs between 100 RA and healthy controls ( $\mathrm{P}=0.188$; Table 2$)$.

\begin{tabular}{|c|c|c|c|}
\hline Genotype & RA & Control & $P$ \\
\hline $920 \mathrm{~A}-1044 \mathrm{C} / 920 \mathrm{~A}-1044 \mathrm{C}$ & $8 / 100$ & 9/100 & \\
\hline $920 \mathrm{~A}-1044 \mathrm{C} / 920 \mathrm{C}-1044 \mathrm{C}$ & $89 / 100$ & $82 / 100$ & 0.188 \\
\hline $920 \mathrm{C}-1044 \mathrm{C} / 920 \mathrm{C}-1044 \mathrm{C}$ & $3 / 100$ & 9/100 & \\
\hline
\end{tabular}

$\mathrm{RA}=$ rheumatoid arthritis. 
The RA samples were stratified according to gender and rheumatoid factor. The stratification results did not reveal any significant differences between the rs 12953258 (-920 C $>$ A) genotype distribution and RA patient subgroups (Table 3). Clinical data indicate both development and severity of RA. Our data suggest that the $S C O S-3$ polymorphism may not affect the increased susceptibility to RA.

\begin{tabular}{|c|c|c|c|c|}
\hline & AA & $\mathrm{AC}$ & $\mathrm{CC}$ & $\mathrm{P}$ \\
\hline Male (N=22) & 2 & 17 & 1 & 0.778 \\
\hline Female $(\mathrm{N}=78)$ & 6 & 72 & 2 & \\
\hline RF- $(\mathrm{N}=18)$ & 0 & 18 & 0 & 0.258 \\
\hline $\mathrm{RF}+(\mathrm{N}=82)$ & 8 & 71 & 3 & \\
\hline
\end{tabular}

\section{DISCUSSION}

Over the last decade, dramatic improvements in the treatment of RA have resulted from increased understanding of the role played by cytokines in the rheumatoid synovium (Edwards et al., 2004; Rahman, 2007). From a functional perspective, SOCS-3 is a convincing candidate gene for genetic association studies investigating susceptibility for RA. Here we studied the association of SOCS-3 sequence variations in RA and related trials in the Chinese population. We identified $-1044 \mathrm{C}>\mathrm{A}$ within the promoter region and rs 12953258 $(-920 \mathrm{C}>\mathrm{A}$ ) in the 5'UTR (exon 2) in SOCS-3 in the samples. Both SNPs were previously described with a minor allele frequency of $8 \%($ rs 12953258) and below $1 \%(-1044 \mathrm{C}>\mathrm{A})$ in Danish individuals (Gylvin et al., 2004). In our study groups comprising 100 RA and 100 healthy controls, there was no mutation at the -1044 site, and $-920 \mathrm{C}>\mathrm{A}$ substitution had an allele frequency of $89 \%$ in RA and $82 \%$ in control. We did not identify sequence variants within the CDS. There was no evidence supporting a role for $-920 \mathrm{C}>\mathrm{A}$ in the etiology of RA (Table 3, P > 0.05). Distribution of these two SNPs in SOCS-3 in a Chinese RA population was quite different from the distribution in the West. Genetic differences between human populations could be one explanation for this.

Gylvin et al. (2004) previously searched for SOCS-3 mutations in Danish subjects and reported that the -920 A-allele deletes an activator protein 2 (AP2) transcription factor binding site. AP2 is involved in angiogenesis, tumor invasion/metastasis and chronic inflammation. Additionally, the TRANSFAC database (GMSTF, 2005) also shows that the rs12953258 C-allele destroys a site bound by ZNF202, a transcriptional repressor that binds to elements found predominantly in genes that participate in lipid metabolism and therefore remains a variant of potential significance in SOCS-3 action in lipid metabolism. There are some studies on the association between rs12953258 and two other SNPs (rs4969169 and rs8064821) in SOCS-3 and obesity, insulin or lipid measures (Jamshidi et al., 2006; Hölter et al., 2007). The studies did not find any association of the rs12953258 alleles with obesity. The lack of association between the SOCS-3 SNPs and obesity is probably because they are not in linkage disequilibrium with a functional site. SNP $-1044 \mathrm{C}>\mathrm{A}$ is located within the proximal STATbinding elements (SBE) where the A-allele destroys the specific palindromic STAT-binding motif TTCCAGGAA resulting in TTACAGAA. Previous studies on the SOCS-3 promoter in- 
dicated that particularly the proximal SBE is functionally relevant for promoter activity (Paul et al., 2000; Gatto et al., 2004). We hypothesized that the $-1044 \mathrm{C}>\mathrm{A}$ variant may result in the loss of an STAT3-binding site and thus influence the activation of SOCS-3. Given the importance of SOCS-3 in RA, it is necessary to know the genetic influences of these polymorphisms of SOCS-3 in RA. We studied the association between rs12953258 and SNP -1044 C>A of SOCS-3 and RA in a Chinese Han population.

Our comparison of 100 RA and 100 healthy controls revealed no evidence of an association with $-1044 \mathrm{C}>\mathrm{A}$ and rs 12953258 of SOCS-3. In our association study, we also compared allele haplotype and genotype frequencies of $-1044 \mathrm{C}>\mathrm{A}$ and rs 12953258 in RA. However, no evidence of an association with RA was detected. Because of the potential functional role of these SNPs and their infrequency, we additionally performed linkage disequilibrium analyses in this subject. The test revealed no evidence of transmission disequilibrium.

Some shortcomings of this study should be mentioned. The association study did not find any effects within the region of SOCS-3 with regard to RA. Given the existing data, the study may be underpowered to demonstrate small associations. Aiming to investigate these smaller effects, the data presented may be valuable for future meta-analyses in combination with additional future available data. The existing data did not investigate the potential interaction with environmental factors such as smoking. These interactions are unlikely to exist given our data. However, the analysis of interactions further reduces the power of studies making future meta-analyses also desirable.

In conclusion, our results do not suggest a major role for the SNPs analyzed in SOCS3 in RA among a Chinese population. However, the relevance of this variation in gene regulation warrants functional studies. Since RA is viewed as a polygenic disorder, products of numerous genes may be involved. To exclude moderate effects of the investigated sequence variations in RA, large samples need to be assessed.

\section{ACKNOWLEDGMENTS}

Research supported by grants from the Scientific Research Fund of the Ministry of Health of China (\#WKJ2007-3-001).

\section{REFERENCES}

Alexander WS (2002). Suppressors of cytokine signalling (SOCS) in the immune system. Nat. Rev. Immunol. 2: 410-416. Arnett FC, Edworthy SM, Bloch DA, McShane DJ, et al. (1988). The American Rheumatism Association 1987 revised criteria for the classification of rheumatoid arthritis. Arthritis Rheum. 31: 315-324.

Edwards JC, Szczepanski L, Szechinski J, Filipowicz-Sosnowska A, et al. (2004). Efficacy of B-cell-targeted therapy with rituximab in patients with rheumatoid arthritis. N. Engl. J. Med. 350: 2572-2581.

Egan PJ, Lawlor KE, Alexander WS and Wicks IP (2003). Suppressor of cytokine signaling-1 regulates acute inflammatory arthritis and T cell activation. J. Clin. Invest. 111: 915-924.

Firestein GS and Zvaifler NJ (1997). Anticytokine therapy in rheumatoid arthritis. N. Engl. J. Med. 337: 195-197.

Gatto L, Berlato C, Poli V, Tininini S, et al. (2004). Analysis of SOCS-3 promoter responses to interferon gamma. J. Biol. Chem. 279: 13746-13754.

GMSTF (2005). Genomatix MatInspector Search for Transcription Factor Binding Sites. Available at [http://www. genomatix.de/online_help/help_matinspector/matinspector_help.html]. Acessed December 10, 2009.

Gylvin T, Nolsoe R, Hansen T, Nielsen EM, et al. (2004). Mutation analysis of suppressor of cytokine signalling 3, a candidate gene in type 1 diabetes and insulin sensitivity. Diabetologia 47: 1273-1277.

Hölter K, Wermter AK, Scherag A, Siegfried W, et al. (2007). Analysis of sequence variations in the suppressor of 
cytokine signaling (SOCS)-3 gene in extremely obese children and adolescents. BMC Med. Genet. 8: 21.

Isomaki P, Alanara T, Isohanni P, Lagerstedt A, et al. (2007). The expression of SOCS is altered in rheumatoid arthritis. Rheumatology 46: 1538-1546.

Jamshidi Y, Snieder H, Wang X, Spector TD, et al. (2006). Common polymorphisms in SOCS3 are not associated with body weight, insulin sensitivity or lipid profile in normal female twins. Diabetologia 49: 306-310.

Maini R, St Clair EW, Breedveld F, Furst D, et al. (1999). Infliximab (chimeric anti-tumour necrosis factor alpha monoclonal antibody) versus placebo in rheumatoid arthritis patients receiving concomitant methotrexate: a randomised phase III trial. ATTRACT Study Group. Lancet 354: 1932-1939.

Paul C, Seiliez I, Thissen JP and Le Cam A (2000). Regulation of expression of the rat SOCS-3 gene in hepatocytes by growth hormone, interleukin-6 and glucocorticoids mRNA analysis and promoter characterization. Eur. J. Biochem. 267: 5849-5857.

Rahman A (2007). Regulators of cytokine signalling in rheumatoid arthritis. Rheumatology 46: 1745-1746.

Rottapel R (2001). Putting the brakes on arthritis: can suppressors of cytokine signaling (SOCS) suppress rheumatoid arthritis? J. Clin. Invest. 108: 1745-1747.

Shi YY and He L (2005). SHEsis, a powerful software platform for analyses of linkage disequilibrium, haplotype construction, and genetic association at polymorphism loci. Cell Res. 15: 97-98.

Shouda T, Yoshida T, Hanada T, Wakioka T, et al. (2001). Induction of the cytokine signal regulator SOCS3/CIS3 as a therapeutic strategy for treating inflammatory arthritis. J. Clin. Invest. 108: 1781-1788.

Smolen JS and Steiner G (2003). Therapeutic strategies for rheumatoid arthritis. Nat. Rev. Drug Discov. 2: 473-488.

Starr R, Willson TA, Viney EM, Murray LJ, et al. (1997). A family of cytokine-inducible inhibitors of signalling. Nature 387: 917-921.

Weinblatt ME, Kremer JM, Bankhurst AD, Bulpitt KJ, et al. (1999). A trial of etanercept, a recombinant tumor necrosis factor receptor:Fc fusion protein, in patients with rheumatoid arthritis receiving methotrexate. N. Engl. J. Med. 340: 253-259.

Weinblatt ME, Keystone EC, Furst DE, Moreland LW, et al. (2003). Adalimumab, a fully human anti-tumor necrosis factor alpha monoclonal antibody, for the treatment of rheumatoid arthritis in patients taking concomitant methotrexate: the ARMADA trial. Arthritis Rheum. 48: 35-45.

Wong PK, Egan PJ, Croker BA, O’Donnell K, et al. (2006). SOCS-3 negatively regulates innate and adaptive immune mechanisms in acute IL-1-dependent inflammatory arthritis. J. Clin. Invest. 116: 1571-1581. 